\title{
Can Quantum-Mechanical Description of Physical Reality be Considered Complete?
}

\author{
N. BoHr, Institute for Theoretical Physics, University, Copenhagen
}

(Received July 13, 1935)

\begin{abstract}
It is shown that a certain "criterion of physical reality" formulated in a recent article with the above title by A. Einstein, B. Podolsky and N. Rosen contains an essential ambiguity when it is applied to quantum phenomena. In this connection a viewpoint termed "complementarity" is explained from which quantum-mechanical description of physical phenomena would seem to fulfill, within its scope, all rational demands of completeness.
\end{abstract}

$I^{\mathrm{N}}$ $\mathrm{N}$ a recent article $^{1}$ under the above title $\mathrm{A}$. Einstein, B. Podolsky and N. Rosen have presented arguments which lead them to answer the question at issue in the negative. The trend of their argumentation, however, does not seem to me adequately to meet the actual situation with which we are faced in atomic physics. I shall therefore be glad to use this opportunity to explain in somewhat greater detail a general viewpoint, conveniently termed "complementarity," which I have indicated on various previous occasions, ${ }^{2}$ and from which quantum mechanics within its scope would appear as a completely rational description of physical phenomena, such as we meet in atomic processes.

The extent to which an unambiguous meaning can be attributed to such an expression as "physical reality" cannot of course be deduced from a priori philosophical conceptions, but-as the authors of the article cited themselves emphasize - must be founded on a direct appeal to experiments and measurements. For this purpose they propose a "criterion of reality" formulated as follows: "If, without in any way disturbing a system, we can predict with certainty the value of a physical quantity, then there exists an element of physical reality corresponding to this physical quantity." By means of an interesting example, to which we shall return below, they next proceed to show that in quantum mechanics, just as in classical mechanics, it is possible under suitable conditions to predict the value of any given variable pertaining to the description of a mechanical system from measurements performed entirely on other systems which previously have been in

${ }^{1}$ A. Einstein, B. Podolsky and N. Rosen, Phys. Rev. 47, 777 (1935).

${ }^{2}$ Cf. N. Bohr, Atomic Theory and Description of Nature, I (Cambridge, 1934). interaction with the system under investigation. According to their criterion the authors therefore want to ascribe an element of reality to each of the quantities represented by such variables. Since, moreover, it is a well-known feature of the present formalism of quantum mechanics that it is never possible, in the description of the state of a mechanical system, to attach definite values to both of two canonically conjugate variables, they consequently deem this formalism to be incomplete, and express the belief that a more satisfactory theory can be developed.

Such an argumentation, however, would hardly seem suited to affect the soundness of quantum-mechanical description, which is based on a coherent mathematical formalism covering automatically any procedure of measurement like that indicated.* The apparent contradiction in

* The deductions contained in the article cited may in this respect be considered as an immediate consequence of the transformation theorems of quantum mechanics, which perhaps more than any other feature of the formalism contribute to secure its mathematical completeness and its rational correspondence with classical mechanics. In fact, it is always possible in the description of a mechanical system, consisting of two partial systems (1) and (2), interacting or not, to replace any two pairs of canonically conjugate variables $\left(q_{1} p_{1}\right),\left(q_{2} p_{2}\right)$ pertaining to systems (1) and (2), respectively, and satisfying the usual commutation rules

$$
\begin{gathered}
{\left[q_{1} p_{1}\right]=\left[q_{2} p_{2}\right]=i h / 2 \pi,} \\
{\left[q_{1} q_{2}\right]=\left[p_{1} p_{2}\right]=\left[q_{1} p_{2}\right]=\left[q_{2} p_{1}\right]=0,}
\end{gathered}
$$

by two pairs of new conjugate variables $\left(Q_{1} P_{1}\right),\left(Q_{2} P_{2}\right)$ related to the first variables by a simple orthogonal transformation, corresponding to a rotation of angle $\theta$ in the planes $\left(q_{1} q_{2}\right),\left(p_{1} p_{2}\right)$

$$
\begin{array}{ll}
q_{1}=Q_{1} \cos \theta-Q_{2} \sin \theta & p_{1}=P_{1} \cos \theta-P_{2} \sin \theta \\
q_{2}=Q_{1} \sin \theta+Q_{2} \cos \theta & p_{2}=P_{1} \sin \theta+P_{2} \cos \theta
\end{array}
$$

Since these variables will satisfy analogous commutation rules, in particular

$$
\left[Q_{1} P_{1}\right]=i h / 2 \pi, \quad\left[Q_{1} P_{2}\right]=0,
$$

it follows that in the description of the state of the combined system definite numerical values may not be assigned to both $Q_{1}$ and $P_{1}$, but that we may clearly assign 
fact discloses only an essential inadequacy of the customary viewpoint of natural philosophy for a rational account of physical phenomena of the type with which we are concerned in quantum mechanics. Indeed the finite interaction between object and measuring agencies conditioned by the very existence of the quantum of action entails - because of the impossibility of controlling the reaction of the object on the measuring instruments if these are to serve their purpose-the necessity of a final renunciation of the classical ideal of causality and a radical revision of our attitude towards the problem of physical reality. In fact, as we shall see, a criterion of reality like that proposed by the named authors contains-however cautious its formulation may appear-an essential ambiguity when it is applied to the actual problems with which we are here concerned. In order to make the argument to this end as clear as possible, I shall first consider in some detail a few simple examples of measuring arrangements.

Let us begin with the simple case of a particle passing through a slit in a diaphragm, which may form part of some more or less complicated experimental arrangement. Even if the momentum of this particle is completely known before it impinges on the diaphragm, the diffraction by the slit of the plane wave giving the symbolic representation of its state will imply an uncertainty in the momentum of the particle, after it has passed the diaphragm, which is the greater the narrower the slit. Now the width of the slit, at any rate if it is still large compared with the wave-length, may be taken as the uncertainty $\Delta q$ of the position of the particle relative to the diaphragm, in a direction perpendicular to the slit. Moreover, it is simply seen from de Broglie's relation between momentum and wave-length that the uncertainty $\Delta p$ of the momentum of the particle in this direction is correlated to $\Delta q$ by means of Heisenberg's general principle

$$
\Delta p \Delta q \sim h
$$

such values to both $Q_{1}$ and $P_{2}$. In that case it further results from the expressions of these variables in terms of $\left(q_{1} p_{1}\right)$ and $\left(q_{2} p_{2}\right)$, namely

$$
Q_{1}=q_{1} \cos \theta+q_{2} \sin \theta, \quad P_{2}=-p_{1} \sin \theta+p_{2} \cos \theta,
$$

that a subsequent measurement of either $q_{2}$ or $p_{2}$ will allow us to predict the value of $q_{1}$ or $p_{1}$ respectively. which in the quantum-mechanical formalism is a direct consequence of the commutation relation for any pair of conjugate variables. Obviously the uncertainty $\Delta p$ is inseparably connected with the possibility of an exchange of momentum between the particle and the diaphragm; and the question of principal interest for our discussion is now to what extent the momentum thus exchanged can be taken into account in the description of the phenomenon to be studied by the experimental arrangement concerned, of which the passing of the particle through the slit may be considered as the initial stage.

Let us first assume that, corresponding to usual experiments on the remarkable phenomena of electron diffraction, the diaphragm, like the other parts of the apparatus,- - say a second diaphragm . with several slits parallel to the first and a photographic plate,-is rigidly fixed to a support which defines the space frame of reference. Then the momentum exchanged between the particle and the diaphragm will, together with the reaction of the particle on the other bodies, pass into this common support, and we have thus voluntarily cut ourselves off from any possibility of taking these reactions separately into account in predictions regarding the final result of the experiment,- - say the position of the spot produced by the particle on the photographic plate. The impossibility of a closer analysis of the reactions between the particle and the measuring instrument is indeed no peculiarity of the experimental procedure described, but is rather an essential property of any arrangement suited to the study of the phenomena of the type concerned, where we have to do with a feature of individuality completely foreign to classical physics. In fact, any possibility of taking into account the momentum exchanged between the particle and the separate parts of the apparatus would at once permit us to draw conclusions regarding the "course" of such phenomena, - say through what particular slit of the second diaphragm the particle passes on its way to the photographic plate-which would be quite incompatible with the fact that the probability of the particle reaching a given element of area on this plate is determined not by the presence of any particular slit, but by the positions of all the slits of the second diaphragm within reach 
of the associated wave diffracted from the slit of the first diaphragm.

By another experimental arrangement, where the first diaphragm is not rigidly connected with the other parts of the apparatus, it would at least in principle* be possible to measure its momentum with any desired accuracy before and after the passage of the particle, and thus to predict the momentum of the latter after it has passed through the slit. In fact, such measurements of momentum require only an unambiguous application of the classical law of conservation of momentum, applied for instance to a collision process between the diaphragm and some test body, the momentum of which is suitably controlled before and after the collision. It is true that such a control will essentially depend on an examination of the space-time course of some process to which the ideas of classical mechanics can be applied; if, however, all spatial dimensions and time intervals are taken sufficiently large, this involves clearly no limitation as regards the accurate control of the momentum of the test bodies, but only a renunciation as regards the accuracy of the control of their space-time coordination. This last circumstance is in fact quite analogous to the renunciation of the control of the momentum of the fixed diaphragm in the experimental arrangement discussed above, and depends in the last resort on the claim of a purely classical account of the measuring apparatus, which implies the necessity of allowing a latitude corresponding to the quantum-mechanical uncertainty relations in our description of their behavior.

The principal difference between the two experimental arrangements under consideration is, however, that in the arrangement suited for the control of the momentum of the first diaphragm, this body can no longer be used as a measuring instrument for the same purpose as in the previous case, but must, as regards its position relative to the rest of the apparatus, be treated, like the particle traversing the slit, as an object of

* The obvious impossibility of actually carrying out, with the experimental technique at our disposal, such measuring procedures as are discussed here and in the following does clearly not affect the theoretical argument, since the procedures in question are essentially equivalent with atomic processes, like the Compton effect, where a corresponding application of the conservation theorem of momentum is well established. investigation, in the sense that the quantummechanical uncertainty relations regarding its position and momentum must be taken explicitly into account. In fact, even if we knew the position of the diaphragm relative to the space frame before the first measurement of its momentum, and even though its position after the last measurement can be accurately fixed, we lose, on account of the uncontrollable displacement of the diaphragm during each collision process with the test bodies, the knowledge of its position when the particle passed through the slit. The whole arrangement is therefore obviously unsuited to study the same kind of phenomena as in the previous case. In particular it may be shown that, if the momentum of the diaphragm is measured with an accuracy sufficient for allowing definite conclusions regarding the passage of the particle through some selected slit of the second diaphragm, then even the minimum uncertainty of the position of the first diaphragm compatible with such a knowledge will imply the total wiping out of any interference effect-regarding the zones of permitted impact of the particle on the photographic plate - to which the presence of more than one slit in the second diaphragm would give rise in case the positions of all apparatus are fixed relative to each other.

In an arrangement suited for measurements of the momentum of the first diaphragm, it is further clear that even if we have measured this momentum before the passage of the particle through the slit, we are after this passage still left with a free choice whether we wish to know the momentum of the particle or its initial position relative to the rest of the apparatus. In the first eventuality we need only to make a second determination of the momentum of the diaphragm, leaving unknown forever its exact position when the particle passed. In the second eventuality we need only to determine its position relative to the space frame with the inevitable loss of the knowledge of the momentum exchanged between the diaphragm and the particle. If the diaphragm is sufficiently massive in comparison with the particle, we may even arrange the procedure of measurements in such a way that the diaphragm after the first determination of its momentum will remain at rest in some unknown position relative to the 
other parts of the apparatus, and the subsequent fixation of this position may therefore simply consist in establishing a rigid connection between the diaphragm and the common support.

My main purpose in repeating these simple, and in substance well-known considerations, is to emphasize that in the phenomena concerned we are not dealing with an incomplete description characterized by the arbitrary picking out of different elements of physical reality at the cost of sacrifying other such elements, but with a rational discrimination between essentially different experimental arrangements and procedures which are suited either for an unambiguous use of the idea of space location, or for a legitimate application of the conservation theorem of momentum. Any remaining appearance of arbitrariness concerns merely our freedom of handling the measuring instruments, characteristic of the very idea of experiment. In fact, the renunciation in each experimental arrangement of the one or the other of two aspects of the description of physical phenomena,- the combination of which characterizes the method of classical physics, and which therefore in this sense may be considered as complementary to one another,- depends essentially on the impossibility, in the field of quantum theory, of accurately controlling the reaction of the object on the measuring instruments, i.e., the transfer of momentum in case of position measurements, and the displacement in case of momentum measurements. Just in this last respect any comparison between quantum mechanics and ordinary statistical mechanics,- - however useful it may be for the formal presentation of the theory,-is essentially irrelevant. Indeed we have in each experimental arrangement suited for the study of proper quantum phenomena not merely to do with an ignorance of the value of certain physical quantities, but with the impossibility of defining these quantities in an unambiguous way.

The last remarks apply equally well to the special problem treated by Einstein, Podolsky and Rosen, which has been referred to above, and which does not actually involve any greater intricacies than the simple examples discussed above. The particular quantum-mechanical state of two free particles, for which they give an explicit mathematical expression, may be repro- duced, at least in principle, by a simple experimental arrangement, comprising a rigid diaphragm with two parallel slits, which are very narrow compared with their separation, and through each of which one particle with given initial momentum passes independently of the other. If the momentum of this diaphragm is measured accurately before as well as after the passing of the particles, we shall in fact know the sum of the components perpendicular to the slits of the momenta of the two escaping particles, as well as the difference of their initial positional coordinates in the same direction; while of course the conjugate quantities, i.e., the difference of the components of their momenta, and the sum of their positional coordinates, are entirely unknown.* In this arrangement, it is therefore clear that a subsequent single measurement either of the position or of the momentum of one of the particles will automatically determine the position or momentum, respectively, of the other particle with any desired accuracy; at least if the wave-length corresponding to the free motion of each particle is sufficiently short compared with the width of the slits. As pointed out by the named authors, we are therefore faced at this stage with a completely free choice whether we want to determine the one or the other of the latter quantities by a process which does not directly interfere with the particle concerned.

Like the above simple case of the choice between the experimental procedures suited for the prediction of the position or the momentum of a single particle which has passed through a slit in a diaphragm, we are, in the "freedom of choice" offered by the last arrangement, just concerned with a discrimination between differen experimental procedures which allow of the unambiguous use of complementary classical concepts. In fact to measure the position of one of the particles can mean nothing else than to establish a correlation between its behavior and some

\footnotetext{
* As will be seen, this description, apart from a trivial normalizing factor, corresponds exactly to the transformation of variables described in the preceding footnote if $\left(q_{1} p_{1}\right),\left(q_{2} p_{2}\right)$ represent the positional coordinates and components of momenta of the two particles and if $\theta=-\pi / 4$. It may also be remarked that the wave function given by formula (9) of the article cited corresponds to the special choice of $P_{2}=0$ and the limiting case of two infinitely narrow slits.
} 
instrument rigidly fixed to the support which defines the space frame of reference. Under the experimental conditions described such a measurement will therefore also provide us with the knowledge of the location, otherwise completely unknown, of the diaphragm with respect to this space frame when the particles passed through the slits. Indeed, only in this way we obtain a basis for conclusions about the initial position of the other particle relative to the rest of the apparatus. By allowing an essentially uncontrollable momentum to pass from the first particle into the mentioned support, however, we have by this procedure cut ourselves off from any future possibility of applying the law of conservation of momentum to the system consisting of the diaphragm and the two particles and therefore have lost our only basis for an unambiguous application of the idea of momentum in predictions regarding the behavior of the second particle. Conversely, if we choose to measure the momentum of one of the particles, we lose through the uncontrollable displacement inevitable in such a measurement any possibility of deducing from the behavior of this particle the position of the diaphragm relative to the rest of the apparatus, and have thus no basis whatever for predictions regarding the location of the other particle.

From our point of view we now see that the wording of the above-mentioned criterion of physical reality proposed by Einstein, Podolsky and Rosen contains an ambiguity as regards the meaning of the expression "without in any way disturbing a system." Of course there is in a case like that just considered no question of a mechanical disturbance of the system under investigation during the last critical stage of the measuring procedure. But even at this stage there is essentially the question of an influence on the very conditions which define the possible types of predictions regarding the future behavior of the system. Since these conditions constitute an inherent element of the description of any phenomenon to which the term "physical reality" can be properly attached, we see that the argumentation of the mentioned authors does not justify their conclusion that quantum-mechanical description is essentially incomplete. On the contrary this description, as appears from the pre- ceding discussion, may be characterized as a rational utilization of all possibilities of unambiguous interpretation of measurements, compatible with the finite and uncontrollable interaction between the objects and the measuring instruments in the field of quantum theory. In fact, it is only the mutual exclusion of any two experi. mental procedures, permitting the unambiguous definition of complementary physical quantities, which provides room for new physical laws, the coexistence of which might at first sight appear irreconcilable with the basic principles of science. It is just this entirely new situation as regards the description of physical phenomena, that the notion of complementarity aims at characterizing.

The experimental arrangements hitherto discussed present a special simplicity on account of the secondary role which the idea of time plays in the description of the phenomena in question. It is true that we have freely made use of such words as "before" and "after" implying timerelationships; but in each case allowance must be made for a certain inaccuracy, which is of no importance, however, so long as the time intervals concerned are sufficiently large compared with the proper periods entering in the closer analysis of the phenomenon under investigation. As soon as we attempt a more accurate time description of quantum phenomena, we meet with well-known new paradoxes, for the elucidation of which further features of the interaction between the objects and the measuring instruments must be taken into account. In fact, in such phenomena we have no longer to do with experimental arrangements consisting of apparatus essentially at rest relative to one another, but with arrangements containing moving parts,-like shutters before the slits of the diaphragms, - controlled by mechanisms serving as clocks. Besides the transfer of momentum, discussed above, between the object and the bodies defining the space frame, we shall therefore, in such arrangements, have to consider an eventual exchange of energy between the object and these clock-like mechanisms.

The decisive point as regards time measurements in quantum theory is now completely analogous to the argument concerning measurements of positions outlined above. Just as the transfer of momentum to the separate parts of 
the apparatus, - the knowledge of the relative positions of which is required for the description of the phenomenon, - has been seen to be entirely uncontrollable, so the exchange of energy between the object and the various bodies, whose relative motion must be known for the intended use of the apparatus, will defy any closer analysis. Indeed, it is excluded in principle to control the energy which goes into the clocks without interfering essentially with their use as time indicators. This use in fact entirely relies on the assumed possibility of accounting for the functioning of each clock as well as for its eventual comparison with other clocks on the basis of the methods of classical physics. In this account we must therefore obviously allow for a latitude in the energy balance, corresponding to the quantum-mechanical uncertainty relation for the conjugate time and energy variables. Just as in the question discussed above of the mutually exclusive character of any unambiguous use in quantum theory of the concepts of position and momentum, it is in the last resort this circumstance which entails the complementary relationship between any detailed time account of atomic phenomena on the one hand and the unclassical features of intrinsic stability of atoms, disclosed by the study of energy transfers in atomic reactions on the other hand.

This necessity of discriminating in each experimental arrangement between those parts of the physical system considered which are to be treated as measuring instruments and those which constitute the objects under investigation may indeed be said to form a principal distinction between classical and quantum-mechanical description of physical phenomena. It is true that the place within each measuring procedure where this discrimination is made is in both cases largely a matter of convenience. While, however, in classical physics the distinction between object and measuring agéncies does not entail any difference in the character of the description of the phenomena concerned, its fundamental importance in quantum theory, as we have seen, has its root in the indispensable use of classical concepts in the interpretation of all proper measurements, even though the classical theories do not suffice in accounting for the new types of regularities with which we are concerned in atomic physics.
In accordance with this situation there can be no question of any unambiguous interpretation of the symbols of quantum mechanics other than that embodied in the well-known rules which allow to predict the results to be obtained by a given experimental arrangement described in a totally classical way, and which have found their general expression through the transformation theorems, already referred to. By securing its proper correspondence with the classical theory, these theorems exclude in particular any imaginable inconsistency in the quantum-mechanical description, connected with a change of the place where the discrimination is made between object and measuring agencies. In fact it is an obvious consequence of the above argumentation that in each experimental arrangement and measuring procedure we have only a free choice of this place within a region where the quantum-mechanical description of the process concerned is effectively equivalent with the classical description.

Before concluding I should still like to emphasize the bearing of the great lesson derived from general relativity theory upon the question of physical reality in the field of quantum theory. In fact, notwithstanding all characteristic differences, the situations we are concerned with in these generalizations of classical theory present striking analogies which have often been noted. Especially, the singular position of measuring instruments in the account of quantum phenomena, just discussed, appears closely analogous to the well-known necessity in relativity theory of upholding an ordinary description of all measuring processes, including a sharp distinction between space and time coordinates, although the very essence of this theory is the establishment of new physical laws, in the comprehension of which we must renounce the customary separation of space and time ideas.*

* Just this circumstance, together with the relativistic invariance of the uncertainty relations of quantum mechanics, ensures the compatibility between the argumentation outlined in the present article and all exigencies of relativity theory. This question will be treated in greater detail in a paper under preparation, where the writer will in particular discuss a very interesting paradox suggested by Einstein concerning the application of gravitation theory to energy measurements, and the solution of which offers an especially instructive illustration of the generality of the argument of complementarity. On the same occasion a more thorough discussion of space-time measurements in quantum theory will be given with all necessary mathematical developments and diagrams of experimental 
The dependence on the reference system, in relativity theory, of all readings of scales and clocks may even be compared with the essentially uncontrollable exchange of momentum or energy between the objects of measurements and all instruments defining the space-time system of

arrangements, which had to be left out of this article, where the main stress is laid on the dialectic aspect of the question at issue. reference, which in quantum theory confronts us with the situation characterized by the notion of complementarity. In fact this new feature of natural philosophy means a radical revision of our attitude as regards physical reality, which may be paralleled with the fundamental modification of all ideas regarding the absolute character of physical phenomena, brought about by the general theory of relativity. 